\title{
Application of new ultra-micro automated spectrophotometric determination for serum hydroxybutyrate dehydrogenase activity to diagnosis of myocardial infarction
}

\author{
A. D. Hirst and D. M. Goldberg* \\ From the Department of Chemical Pathology, United Sheffield Hospitals, Sheffield
}

An automated spectrophotometric assay for serum $\alpha$-hydroxybutyrate dehydrogenase (SHBD) activity has been developed. This utilizes the change in absorbance at $340 \mathrm{~nm}$. consequent upon oxidation of NADH. Determinations are made at $38^{\circ} \mathrm{C}$. at a rate of 60 per hour, and measurement of test and blank requires only 15 ulitre serum. Sera of known SHBD activity assayed by a manual kinetic spectrophotometric technique at $25^{\circ} \mathrm{C}$. are used as standards.

The normal range determined for 648 subjects was 40-200 $\mathrm{mIU} / \mathrm{ml}$. There was no difference between the sexes, and in both, SHBD activity increased significantly with age. Sera from 528 patients suspected of having sustained myocardial infarction were assayed. Of 197 patients in whom infarction was established on clinical and electrocardiographic criteria, 150 had raised SHBD activity. Of 286 patients in whom infarction was ultimately excluded, 67 had raised SHBD activity. The accuracy of $S H B D$ activity in confirming the diagnosis of myocardial infarction was comparable with that of aspartate aminotransferase activity as determined spectrophotometrically in the same subjects, and there were advantages in carrying out both procedures. Storage of sera at $-20^{\circ} \mathrm{C}$. resulted in a 15 per cent increase in SHBD activity, whereas a fall of 5 per cent occurred after 7 days at $4^{\circ} \mathrm{C}$.

Human serum contains an enzyme catalysing the reversible reduction of $\alpha$-oxobutyrate according to the following equation:

$\alpha$-oxobutyrate $+\mathrm{NADH}_{2}$

$\rightleftharpoons \alpha$-hydroxybutyrate + NAD

The enzyme concerned has been named serum hydroxybutyrate dehydrogenase (SHBD), but its activity is a function of the lactate dehydrogenase isoenzymes (Rosalki and Wilkinson, 1960). These are composed of two molecular subgroups, and it is the faster-moving isoenzymes rich in the B subgroup that mainly account for SHBD activity (Latner and Skillen, 1968).

The role of SHBD determinations as an aid to the diagnosis of myocardial infarction has been established (Elliott and Wilkinson,

Received 2 June 1969.

*Request for reprints to Dr. D. M. Goldberg, Department of Chemical Pathology, Royal Hospital, Sheffield.
196I ; Elliott, Jepson, and Wilkinson, 1962; Konttinen and Halonen, 1962; Rosalki, 1963; Rosalki and Wilkinson, 1964), though raised levels are known to occur in other conditions, mainly diseases of the liver, megaloblastic anaemias, and the muscular dystrophies (Elliott and Wilkinson, 1961, 1963; Johnston et al., 1966). But the availability of other serum enzyme tests such as aspartate and alanine aminotransferase (SGOT and SGPT) and ATP:creatine phosphotransferase $(\mathrm{CPK})$ has rendered the position of SHBD controversial; some authors consider that its determination in suspected cases of myocardial infarction is most advantageous (Preston, Batsakis, and Briere, 1964; Stuart et al., 1965), while others are of the opinion that it does not contribute more than alternative estimations to diagnostic accuracy in this condition (Nissen, Ranløv, and WeisFogh, 1965; Griffiths, 1966; Smith, 1967). 
In the belief that an automated method for SHBD determination would enable a more rapid and economical throughput of samples, we have devised an ultra-micro automated spectrophotometric procedure for SHBD determination which has many advantages over previous techniques (Schwartz, Kessler, and Bodansky, 196I ; Strandjord and Clayson, I966; Dube, Hunter, and Knight, 1968). This report describes its application to a large series of non-hospitalized control subjects, and to patients in whom the possibility of myocardial infarction was raised by the physician. The diagnostic accuracy of SHBD has been compared with that of the aminotransferases in the latter group.

\section{Material and method}

Apparatus The following items were purchased from Technicon Instruments Co. Ltd., Chertsey, Surrey: proportioning pump module; sampler II module; $120 / \mathrm{hr}$. cam with I : 2 sample: wash ratio; glass coil (105-II73-I) length 1219 $\mathrm{cm}$., $2.0 \mathrm{~mm}$. I.D.

The following items were purchased from Beckman Instruments Ltd., Glenrothes, Fifeshire, Scotland: DB spectrophotometer (Cat. No. Sr $402 \mathrm{H}$ ); 5 " linear/log recorder (Cat. No. S93507H); scale expander (Cat. No. 151060); micro-flowcell (Cat. No. 97290).

\section{Reagents}

(I) $0.067 \mathrm{M}$ phosphate buffer, $\mathrm{pH} 7.40$

This is prepared by dissolving 7.55 g. $\mathrm{Na}_{2} \mathrm{HPO}_{4}$ (anhydrous) and $\mathrm{I} \cdot 8 \mathrm{I} \mathrm{g.} \mathrm{KH}_{2} \mathrm{PO}_{4}$ (anhydrous) in water, adjusting the $p \mathrm{H}$ to 7.40 and the volume to I litre; stable for one week at $4^{\circ} \mathrm{C}$.

\section{(2) Substrate solution}

$2 \mathrm{I} \cdot 0 \mathrm{mg}$. $\beta$-NADH (disodium salt) and $100 \mathrm{mg}$. $\alpha$-oxobutyrate (monosodium salt) are dissolved in roo ml. of above buffer to give final concentrations of $0.3 \mathrm{mM}$ and $8.1 \mathrm{mM}$ respectively. This is sufficient for 70 analyses, and is prepared fresh each day.

\section{(3) Standards}

Chemtrol (Clinton Laboratories, Los Angeles), approximate SHBD activity 900 milli-International Units per $\mathrm{ml}$. ( $\mathrm{mIU} / \mathrm{ml}$.) at $25^{\circ} \mathrm{C}$., was diluted in 0.15 molar $\mathrm{NaCl}$ to give 4 samples covering the range $100-500 \mathrm{mIU} / \mathrm{ml}$. at $25^{\circ} \mathrm{C}$., each being assayed in duplicate by the manual spectrophotometric technique of Rosalki and Wilkinson (1960); they may be kept at $4^{\circ} \mathrm{C}$. for up to one week but must be reassayed each day of use.

Technique The manifold is shown in Fig. $r$. The sampler II is governed by a $120 / \mathrm{hr}$. cam with a sample time of Io seconds and a wash time of 20 seconds. The bubbler consists of an
AO Technicon T-piece with small tygon pump tubing inserted in the side-arm and adjusted to give not less than ro bubbles in ro seconds. The sample stream is introduced through a standard A6 fitting, and equilibrates with the reagent stream in a single mixing coil. The volume of the incubation coil is $35 \mathrm{ml}$. and the incubation time at $38^{\circ} \mathrm{C}$. is approximately to minutes.

The absorbance of the substrate solution at $340 \mathrm{~nm}$. is approximately $\mathrm{r} \cdot 45$. The range-expander is set to give a recorder scale of $0.5-1 \cdot 5$. The system has a range equivalent to SHBD activities of $0-600 \mathrm{mIU} / \mathrm{ml}$. at $25^{\circ} \mathrm{C}$. When sera are pumped with substrate solution, the reduction in absorbance at $340 \mathrm{~nm}$. is proportional to SHBD activity, the latter being determined using a calibration curve drawn from the peaks given by the standards. Blank determinations are carried out by pumping sera with buffer and NADH alone, the final NADH concentration being $0.2 \mathrm{mM}$ to give an absorbance at $340 \mathrm{~nm}$. of approximately $\mathrm{I} \cdot 0$. The effect of serum is to increase the apparent absorbance of the buffered $\mathrm{NADH}$, and this increment, which in clear sera corresponds to about $10 \mathrm{mIU} / \mathrm{ml}$. and in turbid sera to as much as $100 \mathrm{mIU} / \mathrm{ml}$., is converted to SHBD activity from the calibration curve and added to that obtained with the same serum in the presence of substrate solution. The amount of serum sampled is $7.5 \mu \mathrm{l}$. for the test and $7.5 \mu \mathrm{l}$. for the blank.

Carry-over This was assessed by sampling a serum of low activity before and after a serum of high activity (Fig. 2). Nine replicates of the high serum gave a mean of $475 \mathrm{mIU} / \mathrm{ml}$. (SD $5.8 \mathrm{mIU} / \mathrm{ml}$.). Five replicates of the low serum when sampled before the high serum gave a mean of IOI $\mathrm{mIU} / \mathrm{ml}$. (SD 4.2 ) and when sampled after the high serum a mean of $117 \mathrm{mIU} / \mathrm{ml}$. (SD 4.5 ). The carry-over was thus 3.4 per cent of the high-activity serum.

FIG. I Manifold for automated spectrophotometric SHBD determination. Flow rates given as ml./min. SMC represents Single Mixing Coil. AO and A6 are standard Technicon equipment modified only where indicated in text.

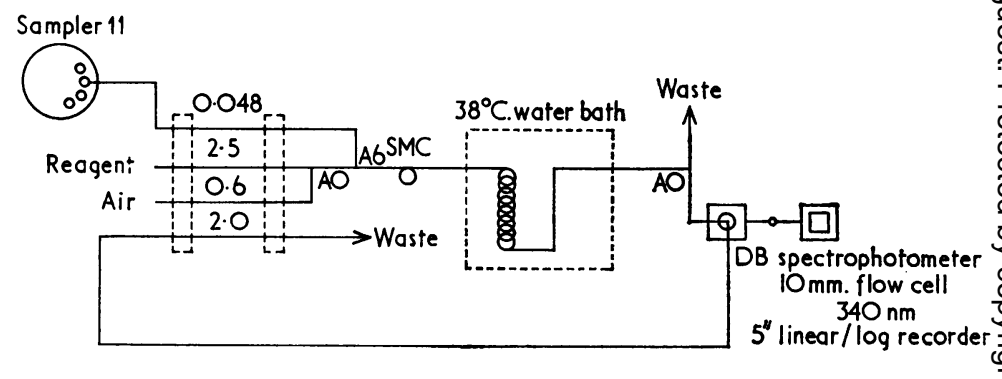




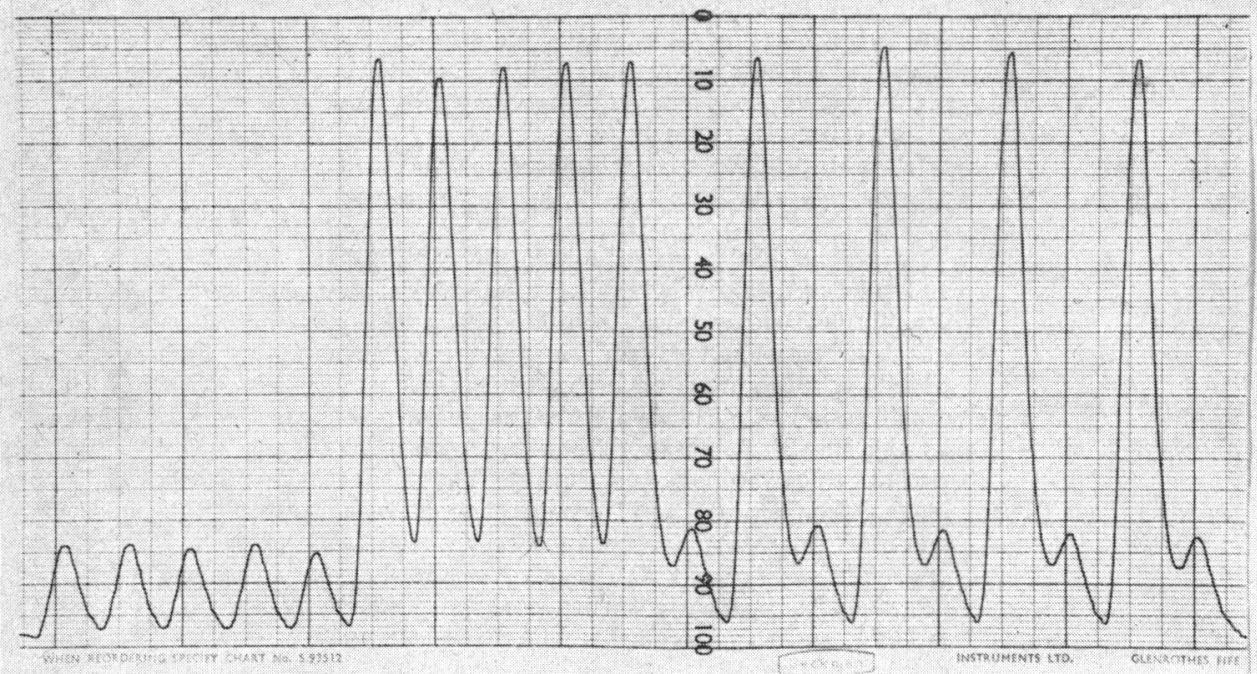

FIG. 2 Reproducibility and carry-over in automated spectrophotometric SHBD determination. Recorder tracing of peaks given by low-activity serum sequentially, high-activity serum sequentially, and the two sera when run alternately.

Comparison of manual and automated method When 36 sera were assayed by both techniques at $38^{\circ} \mathrm{C}$., the automated method gave higher results (mean 15.0 units higher, SD 14.8). This represented 5 per cent of the average activity, and the difference was not statistically significant.

Precision Thirty samples whose HBD activity ranged from II 5 to $705 \mathrm{mIU} / \mathrm{ml}$. were analysed on two independent runs carried out the same day. The average difference between the duplicates was \pm 1.6 per cent and the largest difference in any one pair $\pm 4 \cdot I$ per cent.

Stability One hundred and two samples were analysed fresh, and again 7 days later after storage at $-20^{\circ} \mathrm{C}$. On average, HBD activity rose by 15.4 per cent after storage, 89 samples showing increased activity, 10 decreased activity, and 3 unaltered activity. This rise when evaluated by the paired t-test was highly significant $\left(t_{0}=10.63\right.$; $\mathrm{p}<0.001$ ). Twenty-six samples were analysed fresh, and again after 7 days at $4^{\circ} \mathrm{C}$. A mean fall of 5.0 per cent in HBD activity occurred, 19 samples showing a decrease, 5 an increase, and 2 unaltered activity. This change was also statistically significant $\left(t_{0}=2.3 I ; p<0.05\right)$. It has been established that the slow LDH isoenzymes associated with molecular subgroup A are partially inactivated by freezing (Zondag, 1963; Kreutzer and Fennis, 1964), but enhancement of activity of subgroup B by freezing has not previously been demonstrated.

\section{Clinical material}

Normals Three groups of subjects were studied.

a) Healthy laboratory personnel aged 16 to 37 years sampled in the post-absorptive state between II and 12 a.m. (77 subjects).

b) Subjects attending a well-patient screening clinic aged 18 to 79 years sampled between 2 and 6 p.m. (407 subjects).

c) Ambulant subjects aged 65 and over attending a special screening clinic for the elderly. Many were under treatment for chronic illness but were well compensated at the time of examination. Subjects with ischaemic heart disease and megaloblastic anaemias were excluded. Blood was drawn between 2 and 6 p.m. (I64 subjects).

The total number of subjects was thus 648; the distribution with respect to age and sex is given in Fig. 3. Serum was separated within an hour, divided into aliquots for each subject, and stored at $-20^{\circ} \mathrm{C}$. Over the next 6 weeks, in addition to SHBD activity in all subjects, the following determinations were carried out on subjects in groups a and b: urea, uric acid, cholesterol, alkaline phosphatase, inorganic phosphate, and total protein by Auto Analyser techniques; calcium and magnesium concentration by Atomic Absorption Spectroscopy; the activities of SGOT and SGPT (Henry et al., 1960) 


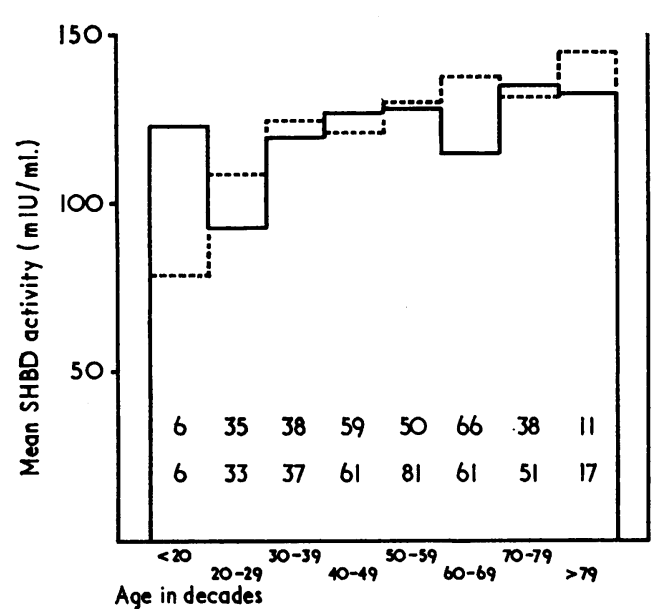

FIG. 3 Change of mean SHBD activity with age. Solid line, men; broken line, women. Inset figures represent total number of men (above) and women (below).

5 ' nucleotidase (Belfield and Goldberg, 1968), and creatine phosphokinase (Worthy, Whitehead, and Goldberg, 1970). The above parameters, together with social class, blood pressure, weight, and percentage overweight (Documenta Geigy, 1956) were studied for a possible influence on SHBD activity using an ICL 1907 computer with a multiple linear correlation programme giving the values and significance of the correlation coefficient $R$.

Ischaemic heart disease The material for this part of the study consisted of sera submitted to the laboratory from patients with suspected myocardial infarction, one sample being taken shortly after admission and a second 24 hours later. A third sample was taken where the results of the first two were ambiguous. The activities of SGOT and SHBD were estimated on all sera. SGPT activity was estimated on the first sample, but not subsequently, unless the initial transaminase ratio raised the possibility of hepatic involvement, i.e. SGPT:SGOT $>$ I. The upper normal limits for SGOT and SGPT in our laboratory are 40 and $30 \mathrm{mIU} / \mathrm{ml}$., respectively.

After six months of operating this system, the entire material (528 cases) was reviewed and, without knowledge of the enzyme results, each subject was assigned to one of the following 4 categories.

\section{(I) Positive}

Serial electrocardiographic examination confirmed the diagnosis of infarction which was supported by convincing clinical evidence, such as characteristic pain, shock, hypotension, and increase in temperature, white cell count, and erythrocyte sedimentation rate.

\section{(2) Electrocardiogram-positive cases without clinical evidence}

Despite unequivocal evidence of recent infarction on the tracings, patients lacked the characteristic pain (silent infarct), and investigation was usually prompted by onset of cardiac failure, arrhythmia, hypotension, or embolism. The precise time of infarction could not always be ascertained.

\section{(3) Clinically-positive cases without confirmation on electrocardiogram}

In these patients, the electrocardiogram gave normal or ambiguous results, often because of previous infarction or the presence of arrhythmia. But the typical clinical features described under (I) above were present, and, despite careful investigation could not be explained on grounds other than infarction.

\section{4) Negative}

Electrocardiograms in these patients gave normal or equivocal results. The clinical features were rarely pathognomonic of infarction and, with few exceptions, were adequately explained by a final diagnosis other than that of myocardial infarction.

\section{Results}

Normals The mean and SD of SHBD activity for men was $128 \pm 35 \mathrm{mIU} / \mathrm{ml}$., and for women $126 \pm 33 \mathrm{mIU} / \mathrm{ml}$. The distribution was typically Gaussian, 13 cases lying above $200 \mathrm{mIU} / \mathrm{ml}$., and 12 below $60 \mathrm{mIU} /$ $\mathrm{ml}$. This range of $60-200 \mathrm{mIU} / \mathrm{ml}$. thus embraced 95 per cent of the population and fulfilled acceptable criteria for a normal range. Though 53 of the 164 subjects in Group C (aged 65 and over) were under treatment for chronic illness, their inclusion had no effect upon the data.

A striking correlation between age and SHBD activity was seen in women (Fig. 3), the mean value almost doubling between the second and ninth decades $(R=0.298 ; \mathrm{p}<$ $0.001)$. In men, the influence of age upon SHBD activity was less apparent $(R=0.227$; $\mathrm{p}<0.005)$. An inverse relation between SHBD activity and serum urea concentration was seen in men $(R=-0.281 ; p<0.001)$, and a direct relation between SHBD activity and serum magnesium concentration in women $(R=0.209 ; p<0.01)$. There was no indication whether the former relation was due to chemical inhibition by urea of LDH isoenzymes (Withycombe, Plummer, and Wilkinson, 1965; Emery, Moores, and Hodson, 1968) or to a dependence of urea concentration and SHBD activity upon renal function. 


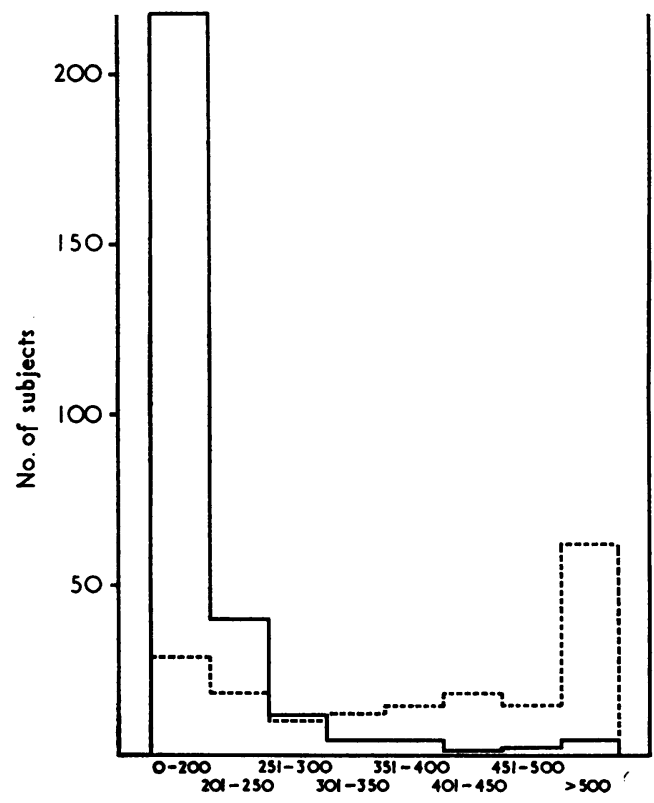

FIG. 4 Distribution of SHBD activity (mIU/ml.) on horizontal axis for positive (broken line) and negative (solid line) cases in suspected myocardial infarction.

Neither explanation clarifies the difference in behaviour seen in both sexes.

Ischaemic heart disease All but 18 of 528 cases investigated were placed in one of the four categories previously defined; those cases defying classification are not considered further.

For the remainder, the highest recorded values for SHBD and SGOT were compared. These are presented for positive and negative cases in Fig. 4 and 5, respectively. Values in excess of 200 and $40 \mathrm{mIU} / \mathrm{ml}$., respectively, were considered raised, and indicative of infarction.

\section{Positive (179 cases)}

Raised SHBD activity was detected in 150 cases and raised SGOT in 144 cases. Since I2 of the cases with raised SGOT activity had even higher SGPT activity in the same specimen, aminotransferase estimations on these subjects ought to be classed as equivocal rather than diagnostic.

Seven cases died within 8 hours of onset of symptoms. Of these, 4 had raised SGOT and only 2 had raised SHBD, emphasizing the more rapid rise of the former after infarction. Fifteen cases were not seen until at

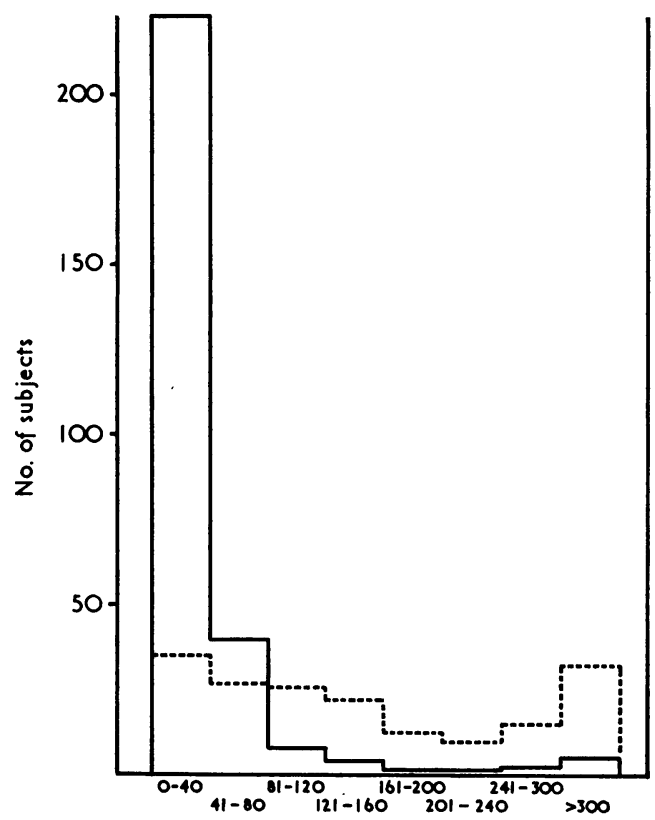

FIG. 5 Distribution of SGOT activity ( $\mathrm{mIU} / \mathrm{ml}$.) on horizontal axis for positive (broken line) and negative (solid line) cases in suspected myocardial infarction.

least 5 days after onset of symptoms. All had normal SGOT activity, but 6 had raised SHBD activity; this confirms the superiority of the latter in detecting late cases of myocardial infarction. Seventeen cases with consistently normal SHBD activity and 16 with consistently normal SGOT activity had at least one sample taken during the 12- to 72-hour period after infarction when maximal enzyme increases are to be expected.

Negative (286 cases)

Raised SHBD activity was detected in 67 cases. Haemolysis of the sample accounted for 12 of these, Addisonian anaemia for 2, pericarditis for 3 , renal failure for 3 , cardiac surgery for 3, non-thoracic major surgery for 4, pulmonary embolism for 3, cardiac arrhythmias for 5, severe angina for 5, congestive cardiac failure for 4 , and bronchial carcinoma for $\mathrm{I}$. The remaining 22 suffered from miscellaneous conditions, mainly abdominal, occasionally associated with raised SHBD activity (Elliott and Wilkinson, 1963).

Of 62 patients with raised SGOT activity, the ratio of aminotransferase activities (SGPT:SGOT > I) in 25 was suggestive of hepatic disease, and the result could not be 
considered as truly false-positive. This left 37 cases in which the increase could be ascribed to haemolysis of the sample in 8, pericarditis in 4, cardiac or major surgery in 4 , congestive cardiac failure in 3 , cardiac arrhythmia in 2, angina and Addisonian anaemia in I case each. A firm diagnosis was not possible in the remaining 14 .

\section{Positive cases on electrocardiogram, without clinical evidence ( 14 cases)}

In 7 , SHBD activity was raised and in 7 it was normal. Two subjects whose infarction occurred more than one week before admission showed raised activity; 2 other subjects in this category had normal activity. SGOT activity was normal in 8 cases and raised in 6 , but in 3 of the latter the SGPT:SGOT ratio was greater than unity and all had congestive heart failure; on this basis alone, infarction would not therefore have been suspected. All 4 cases admitted later than one week from the date of infarction gave normal values for SGOT.

\section{Clinically-positive cases without confirmation on electrocardiogram ( 31 cases)}

In 23, SHBD activity was raised and in 8 it was normal: the latter included one case examined for the first time 16 days after suspected infarction and one case dying within 8 hours of onset of symptoms. SGOT activity was normal in 7 cases and raised in 24, but in 2 of the latter the SGPT:SGOT ratio was greater than unity; aminotransferase determinations in these patients thus did not provide unequivocal support for the clinical diagnosis.

\section{Discussion}

Technical aspects Previous automated determinations of SHBD activity (Schwartz et al., 196r ; Strandjord and Clayson, 1966; Dube et al., 1968) utilize direct calculation of the change in absorbance arising from conversion of NADH to NAD as a function of time for the expression of results. This necessitates accurate knowledge of the incubation time, the path length of the flow cell, and the pumping rates of reagent and sample, some of which may vary from day to day. Absorbance requires to reach a steady plateau, and this may be expensive in terms of both sample and reagent. The assumption is made that there is a linear relation between enzyme activity and the change in absorbance, and this may not be true of high and low activities. The loss of zero-order kinetics is also ignored.
The present method employs as standards sera previously assayed by an accurate kinetic technique for SHBD activity. Pumping rates and incubation time need not be accurately known, and loss of zero-order measurements is compensated as between standards and unknowns. Changes in background reagent-stream absorbance are carefully monitored, and a further standard with every 9 unknowns corrects for possible instrument drift. If serum is aspirated continuously, 85 per cent of the plateau absorbance is reached in Io seconds, 96 per cent in 30 seconds, and 100 per cent in 40 seconds. It is therefore possible to present sera at 30second intervals, sampling for 10 seconds and washing for 20 seconds. This reduces carry-over from sample to sample to approximately 4 per cent, and permits 60 sera with blanks to be assayed per hour, with a total sample volume of only I $5 \mu$ litre for combined test and blank determinations. The speed of sampling is far greater and the volume of sample far smaller than in any previous method, and by carrying out the automated reaction at $38^{\circ} \mathrm{C}$. sensitivity is increased and the relative significance of the blank effectively diminished.

Clinical aspects In this series, estimation of SHBD activity was as reliable as aminotransferase determinations in confirming the diagnosis of myocardial infarction. In many clinical situations, both aminotransferases require to be estimated, especially where possible hepatic involvement is concerned; if this is not done, an unacceptably high falsepositive rate ensues. The choice of a single enzyme determination would thus favour SHBD. The superiority of SHBD in detecting cases presenting several days after the onset of infarction is especially impressive, and confirms previous work (Elliott et al., 1962; Rosalki, 1963; Preston et al., 1964; Stuart et al., 1965). The fact that aminotransferase activities were determined by a kinetic spectrophotometric technique requires emphasis; these have been strongly recommended by Amador, Franey, and Massod (1966) in preference to colorimetric techniques for the diagnosis of infarction, though this position has been disputed by Crowley (1967). Our experience accords with that of Amador et al. (1966). Before undertaking the present work, we surveyed a series of patients classified according to the criteria already outlined in whom 4 consecutive aminotransferase determinations spanning the period of 8-72 hours after onset of symptoms were carried out by a colori- 
metric technique (King, 1958). Only 30 of 46 positive cases gave raised aminotransferase values with a ratio of SGPT:SGOT less than unity; in 5 SGOT was raised but was less than SGPT activity. Seven of 57 negative cases had raised SGOT activity only, and a further Io had increases in both aminotransferases, SGPT being the higher of the two. The results obtained by the present SHBD method have been compared in the Table with those of other authors using various other methods. It is difficult to be sure that the criteria employed by these authors for classifying a patient as positive or negative were the same as ours, or that the timing of blood samples was satisfactory in all cases.

TABLE Percentage of cases positive and negative for myocardial infarction showing raised SHBD activity in present and previous reports

\begin{tabular}{lll}
\hline Authors & $\begin{array}{l}\text { Per cent } \\
\text { positive } \\
\text { cases }\end{array}$ & $\begin{array}{l}\text { Per cent } \\
\text { negative } \\
\text { cases }\end{array}$ \\
\hline Elliott et al. (1962) & 100 & 37 \\
Preston et al. (1964) & 97 & 14 \\
Stuart et al. (1965) & 100 & 63 \\
Griffiths (1966) & 94 & 21 \\
Smith (I967) & 94 & 7 \\
Present & 84 & 23 \\
\hline
\end{tabular}

The percentage figures given for the present series include every positive and negative case, irrespective of factors such as timing of the sample or haemolysis of the specimen which tend to produce unfavourable results. The most fundamental comparison is with the SGOT results in the same series, since the latter estimation was made by the most accurate technique currently available, and both estimations were carried out as part of the routine service of a hospital laboratory. It is clear that SHBD determinations are at least as satisfactory as SGOT determinations in confirming a diagnosis of myocardial infarction, and that there is not total agreement between the results in every case - in other words each of the two estimations has something to offer. It is also apparent that even when extenuating circumstances are taken into account, a significant number of patients give false-negative or false-positive results for one or both determinations. Realization by the clinician that results obtained in a routine service rarely match the enthusiastic claims based upon research studies will do much to ensure the proper assessment of serum enzyme determinations in relation to myocardial infarction.

From the standpoint of fitting SHBD determinations into the work-load of the general laboratory, the present technique has considerable advantages. The samples may be stored at $-20^{\circ} \mathrm{C}$. or at $4^{\circ} \mathrm{C}$. provided that the normal range is adjusted to take account of storage conditions, and all specimens are treated similarly. Micro-tubes containing 20 to $50 \mu \mathrm{l}$. serum are required, thereby minimizing the need for storage space. A week's collection of sera may be analysed in the course of 60 to 90 minutes. The demands on technician time and machine time are minimal, the cost of the reagents modest, and much useful information is available to the clinician, supplementing that obtained from ward procedures, electrocardiography, and aminotransferase estimations. It is in this manner that we recommend the employment of the present technique.

We wish to thank the Medical Staff of the United Sheffield Hospitals who provided blood samples and clinical details of the patients, Dr. Eric Wilkes who provided sera from both screening clinics, and the Technical Staff of the Department of Chemical Pathology who co-operated in this study. We are also indebted to Mr. A. J. Handyside and Mr. K. Trout for help in the computer analysis of the data, the Department of Medical Photography, Royal Hospital, Sheffield, for Fig. I-5, and Dr. Arthur Jordan in whose laboratory much of this work was carried out.

\section{References}

Amador, E., Franey, R. J., and Massod, M. F. (1966). Serum glutamic-oxaloacetic transaminase activity. Diagnostic accuracy of the revised spectrophotometric and the dinitrophenylhydrazine methods. Clinical Chemistry, 12, 475.

Belfield, A., and Goldberg, D. M. (1968). Inhibition of the nucleotidase effect of alkaline phosphatase by $\beta$-glycerophosphate. Nature (London), 219, 73.

Crowley, L. V. (1967). The Reitman-Frankel colorimetric transaminase procedure in suspected myocardial infarction. Clinical Chemistry, 13, 482.

Documenta Geigy (1956). Scientific Tables, 5th ed., p. 255. Basel.

Dube, V., Hunter, D. T., and Knight, J. A. (1968). Automated spectrophotometric assay of serum lactic dehydrogenase and hydroxybutyric dehydrogenase with clinical evaluation. American fournal of Clinical Pathology, 50, 49r.

Elliott, B. A., Jepson, E. M., and Wilkinson, J. H. (1962). Serum $\alpha$-hydroxybutyrate dehydrogenasea new test with improved specificity for myocardial lesions. Clinical Science, 23, 305.

$\longrightarrow$, and Wilkinson, J. H. (I96I). Serum ' $\alpha$-hydroxybutyric dehydrogenase' in myocardial infarction and in liver disease. Lancet, $1,698$.

- , and - (1963). The serum ' $\alpha$-hydroxybutyrate dehydrogenase' in diseases other than myocardial infarction. Clinical Science, 24, 343. 
Emery, A. E. H., Moores, G. E., and Hodson, V. (1968). The determination of lactate dehydrogenase (LDH) isoenzymes with urea. Clinica Chimica Acta, 19, 159.

Griffiths, P. D. (1966). ATP: creatine phosphotransferase in the diagnosis of acute chest pain. British Heart fournal, 28, 199.

Henry, R. J., Chiamori, N., Golub, O. J., and Berkman, S. (1960). Revised spectrophotometric methods for the determination of glutamic-oxalacetic transaminase, glutamic-pyruvic transaminase and lactic acid dehydrogenase. American fournal of Clinical Pathology, 34, 381.

Johnston, H. A., Wilkinson, J. H., Withycombe, W. A., and Raymond, S. (1966). Alpha-hydroxybutyrate dehydrogenase activity in sex-linked muscular dystrophy. Fournal of Clinical Pathology, 19, 250.

King, J. (1958). Routine methods for the estimation of serum transaminase. Fournal of Medical Laboratory Technology, 15, 17.

Konttinen, A., and Halonen, P. I. (1962). Serum $\alpha$-hydroxybutyric dehydrogenase (HBD) in myocardial infarction. Comparison with glutamic oxalacetic transaminase (GOT) and lactic dehydrogenase $(\mathrm{LDH})$. American fournal of Cardiology, 10, 525 .

Kreutzer, H. H., and Fennis, W. H. S. (1964). Lactic dehydrogenase isoenzymes in blood serum after storage at different temperatures. Clinica Chimica Acta, 9, 64 .

Latner, A. L., and Skillen, A. W. (1968). Isoenzymes in Biology and Medicine. Academic Press, London and New York.

Nissen, N. I., Ranløv, P., and Weis-Fogh, J. (1965). Evaluation of four different serum enzymes in the diagnosis of acute myocardial infarction. British Heart fournal, 27, 520.

Preston, J. A., Batsakis, J. G., and Briere, R. O. (1964). Serum ' $\alpha$-hydroxybutyrate dehydrogenase'. A clinical and laboratory evaluation in patients with myocardial infarct. American fournal of Clinical Pathology, 4r, 237.

Rosalki, S. B. (1963). Serum $\alpha$-hydroxybutyrate dehydrogenase: a new test for myocardial infarction. British Heart fournal, 25, 795.

$\longrightarrow$, and Wilkinson, J. H. (1960). Reduction of $\alpha$ ketobutyrate by human serum. Nature (London), 188, I I I0.

$\longrightarrow$, and - (1964). Serum alpha-hydroxybutyrate dehydrogenase in diagnosis. Fournal of the American Medical Association, 189, 61.

Schwartz, M. K., Kessler, G., and Bodansky, $O$. (196I). Automated assay of activities of enzymes involving the diphosphopyridine nucleotide $\rightleftharpoons$ reduced diphosphopyridine nucleotide reaction. fournal of Biological Chemistry, 236, 1207.

Smith, A. F. (1967). Diagnostic value of serum-creatine-kinase in a coronary care unit. Lancet, 2,178 .

Strandjord, P. E., and Clayson, K. J. (1966). An automated method for the determination of total lactate dehydrogenase, heat-stable lactate dehydrogenase isozyme-I, and hydroxybutyrate dehydrogenase. Fournal of Laboratory and Clinical Medicine, 67, 131 .

Stuart, J., Crawford, I. C., Forshall, J., and Owen, J. A. (1965). Serum hydroxybutyrate dehydrogenase in myocardial infarction. British Medical fournal, $\mathbf{I}$, 423.

Withycombe, W. A., Plummer, D. T., and Wilkinson, J. H. (1965). Organ specificity and lactate-dehydrogenase activity. Differential inhibition by urea and related compounds. Biochemical fournal, 94, 384.

Worthy, E., Whitehead, P., and Goldberg, D. M. (1970). Advantages and limitations of a simplified colorimetric assay for ATP: creatine phosphotransferase activity of human serum. Enzymologia Biologica et Clinica (Basel), II 193.

Zondag, H. A. (1963). Lactate dehydrogenase isozymes: lability at low temperature. Science, 142, 965. 